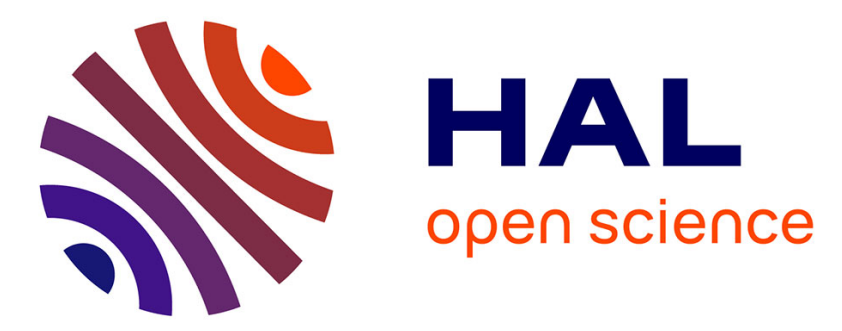

\title{
Suivi in situ de l'avancement du frittage d'une poudre d'acier
}

O. Lame, Sylvie Bourdineaud-Bordère, Dominique Denux, Didier Bouvard

\section{To cite this version:}

O. Lame, Sylvie Bourdineaud-Bordère, Dominique Denux, Didier Bouvard. Suivi in situ de l'avancement du frittage d'une poudre d'acier. Journal de Physique IV Proceedings, 2002, 12 (11), pp.377-384. 10.1051/jp4:20020515 . hal-00702571

\section{HAL Id: hal-00702571 \\ https://hal.science/hal-00702571}

Submitted on 30 May 2012

HAL is a multi-disciplinary open access archive for the deposit and dissemination of scientific research documents, whether they are published or not. The documents may come from teaching and research institutions in France or abroad, or from public or private research centers.
L'archive ouverte pluridisciplinaire HAL, est destinée au dépôt et à la diffusion de documents scientifiques de niveau recherche, publiés ou non, émanant des établissements d'enseignement et de recherche français ou étrangers, des laboratoires publics ou privés. 


\title{
Suivi in situ de l'avancement du frittage d'une poudre d'acier
}

\author{
O. Lame, S. Bordère', D. Denux ${ }^{1}$ et D. Bouvard
}

Laboratoire Génie Physique et Mécanique des Matériaux, INPG, UMR 5010 du CNRS, BP. 46, 38402 Saint-Martin-d'Hères cedex, France

${ }^{1}$ Institut de Chimie de la Matière Condensée de Bordeaux, 87 avenue du Docteur Schweitzer, 33608 Pessac cedex, France

Résumé . Une manière simple de caractériser l'avancement du frittage d'une poudre est de suivre ses variations de densité relative. Cependant, pour les poudres d'acier préalablement compactées en matrice, la densité initiale est grande, par conséquent évolue peu lors du frittage. Au contraire, la microstructure et les propriétés du matériau évoluent beaucoup. Dans cette étude, l'avancement du frittage est suivi in situ au cours du frittage par deux caractéristiques physiques : la résistivité électrique et la viscosité. Les techniques innovantes développées pour ces mesures expérimentales sont présentées ainsi que l'intérêt des résultats pour la compréhension et la modélisation du frittage.

\section{INTRODUCTION}

La représentation macroscopique de l'avancement du frittage d'une poudre est généralement définie par l'évolution de sa densité relative. Ce paramètre est particulièrement attractif pour beaucoup de poudres, en particulier les poudres " dures ». En effet, ces dernières présentent généralement de faibles densités relatives (de l'ordre de 0.5) après compaction. Lors du frittage, le retrait est important et la densité relative peut se rapprocher de 1 . Les propriétés physiques et mécaniques du matériau sont fortement corrélées à la densité relative. Ainsi, dans le cadre d'une modélisation macroscopique du frittage dans le cadre de la mécanique des milieux continus, la densité relative est retenue comme unique variable interne, permettant de décrire les évolutions irréversibles survenant tout au long du frittage. De nombreuses simulations numériques fondées sur cette hypothèse ont montré la pertinence d'une telle approche [1-4].

Concernant les poudres ductiles, la densité relative n'est plus un bon paramètre. En effet, lors de la compaction en matrice, les particules de poudres se déforment plastiquement et la densité relative peut atteindre des valeurs supérieures à $90 \%$. Par la suite le frittage ne modifie plus significativement la densité alors que les propriétés du matériau évoluent considérablement. C'est le cas de la poudre que nous étudions ici : la poudre d'acier Distaloy AE. Pour ce matériau, de nouveaux paramètres doivent être définis pour caractériser les évolutions irréversibles induites par le frittage.

En utilisant la procédure expérimentale définie par Cai et al. [5], plusieurs auteurs ont pu mesurer la viscosité d'un matériau continûment pendant le frittage. En particulier, Lame et al. ont montré les fortes évolutions de la viscosité du Distaloy en cours de frittage [6]. Par ailleurs, Simchi, Danninger et al. $[7,8,5]$ ont mesuré la conductivité électrique d'une poudre à base de Fer à différents stades de frittage. Ils ont montré que ce paramètre est représentatif de l'évolution de la microstructure en cours de frittage

La présente étude porte à la fois sur la viscosité et la résistivité électrique du Distaloy AE. Les évolutions de ces deux paramètres sont suivies in situ pendant le frittage. Un dilatomètre a été spécialement équipé pour permettre la mesure simultanée du retrait et la résistivité électrique d'un matériau au cours d'un cycle thermique. La viscosité est quant à elle déduite d'essais de flexion réalisés dans un dilatomètre permettant d'appliquer de faibles charges. Grâce aux données recueillies lors de ces deux expériences, deux variables d'avancement du frittage ont été définies. Leurs évolutions ont été analysées dans l'optique d'une amélioration de la compréhension et de la modélisation du frittage des poudres métalliques. 


\section{MESURES DE RETRAIT ET DE RESISTIVITE}

\subsection{Equipement}

Le dispositif expérimental développé pour la mesure simultanée de retrait et résistivité électrique (figure 1) est basé sur un dilatomètre différentiel de marque Netzsch. La mesure du retrait se fait par la comparaison du déplacement de l'échantillon avec un échantillon de référence en alumine. La mesure de résistivité est réalisée grâce à un dispositif de mesure « 4 pointes » [10]. La résistivité, $\rho$, est déduite de la mesure de la tension U induite par l'application d'une intensité 1 constante :

Où $\mathrm{S}$ est la surface de la section perpendiculaire aux lignes de courrant et $\mathrm{L}$ est la distance entre les deux fils de tension. Un nano-voltmètre est utilisé pour obtenir une bonne précision de mesure. En disposant les fils de tension et d'intensité sur l'une des faces de l'échantillon parallèlement à l'axe de compression, la résistivité "axiale" est mesurée. Lorsque les fils sont disposés perpendiculairement à l'axe de compression, la résistivité "transverse" est mesurée.

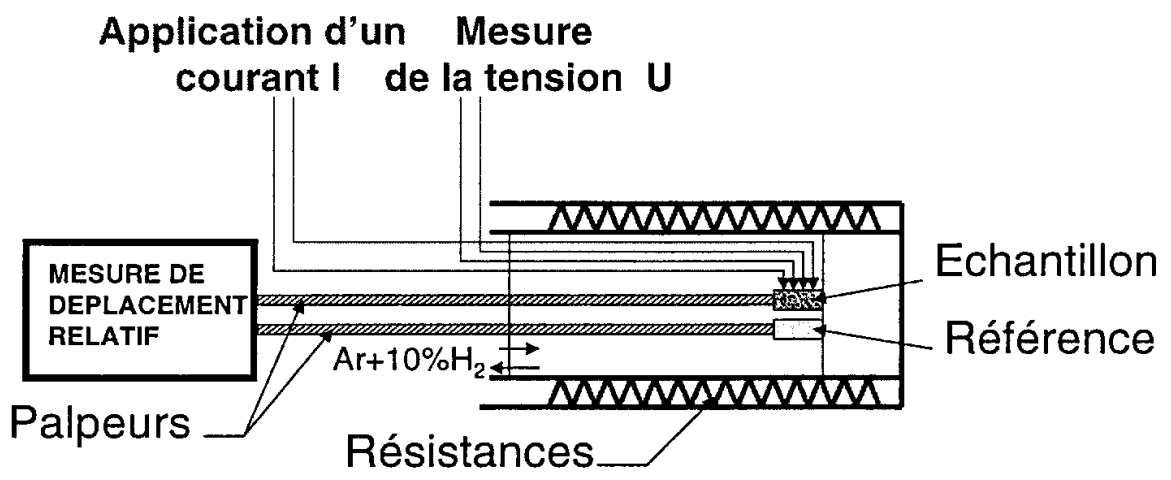

Figure 1 : Schéma du dilatomètre avec mesure de résistivité.

\subsection{Résultats}

Les échantillons sont obtenus à partir d'une poudre de Distaloy $\mathrm{AE}(\mathrm{Fe}+1,5 \% \mathrm{Cu},+0,5 \%$ Mo et $4 \%$ Ni) mélangée avec $0,6 \%$ de graphite en poids. La poudre est compactée en matrice jusqu'à des pressions de $750 \mathrm{MPa}$ et $400 \mathrm{MPa}$. La densité des échantillons obtenus est respectivement de $7,08 \mathrm{~g} / \mathrm{cm}^{3}$ et 6,5 $\mathrm{g} / \mathrm{cm}^{3} \pm 0,05 \mathrm{~g} / \mathrm{cm}^{3}$. Les échantillons sont ensuite frittés sous atmosphère réductrice $\mathrm{Ar}+10 \% \mathrm{H}_{2}$. Un premier cycle est appliqué comportant une rampe à $10^{\circ} \mathrm{C} / \mathrm{min}$, un palier de deux heures à $1130^{\circ} \mathrm{C}$ puis un refroidissement à $10^{\circ} \mathrm{C} / \mathrm{min}$. Sur la figure 2 sont reportés les résultats pour 2 échantillons (parallélépipèdes de $8 \times 8 \times 4 \mathrm{~mm}$ ) à $6.5 \mathrm{~g} / \mathrm{cm}^{3}$. Le premier est mesuré dans le sens axial et le second dans le sens transverse. La première partie de la courbe correspond à la dilatation thermique de l'échantillon. Durant cette période et jusqu'à $800^{\circ} \mathrm{C}$, plusieurs phénomènes, comme le déliantage ou la relaxation de contraintes résiduelles apparaissent. Les petites perturbations mises en évidences par des flèches sur les courbes correspondent vraisemblablement à ces effets. La transformation de phase $\alpha \rightarrow \gamma$ intervient entre 800 et $900^{\circ} \mathrm{C}$. Cette transformation est certainement bien plus complexe que la transformation classique $\mathrm{du}$ fer dense. Pendant cette étape une anisotropie en déformation apparaît. Pendant le palier, un retrait égal dans les deux directions est observé. Après refroidissement, le retrait est d'environ $0,9 \%$ dans la direction de compaction et $0,75 \%$ dans la direction transverse. Immédiatement à la suite de ce cycle, un 
second cycle est appliqué comportant un palier à $1130^{\circ} \mathrm{C}$ de seulement quelques minutes. On observe une déformation isotrope. L'amplitude de la déformation lors de la transformation de phase est bien plus faible que lors du premier cycle.

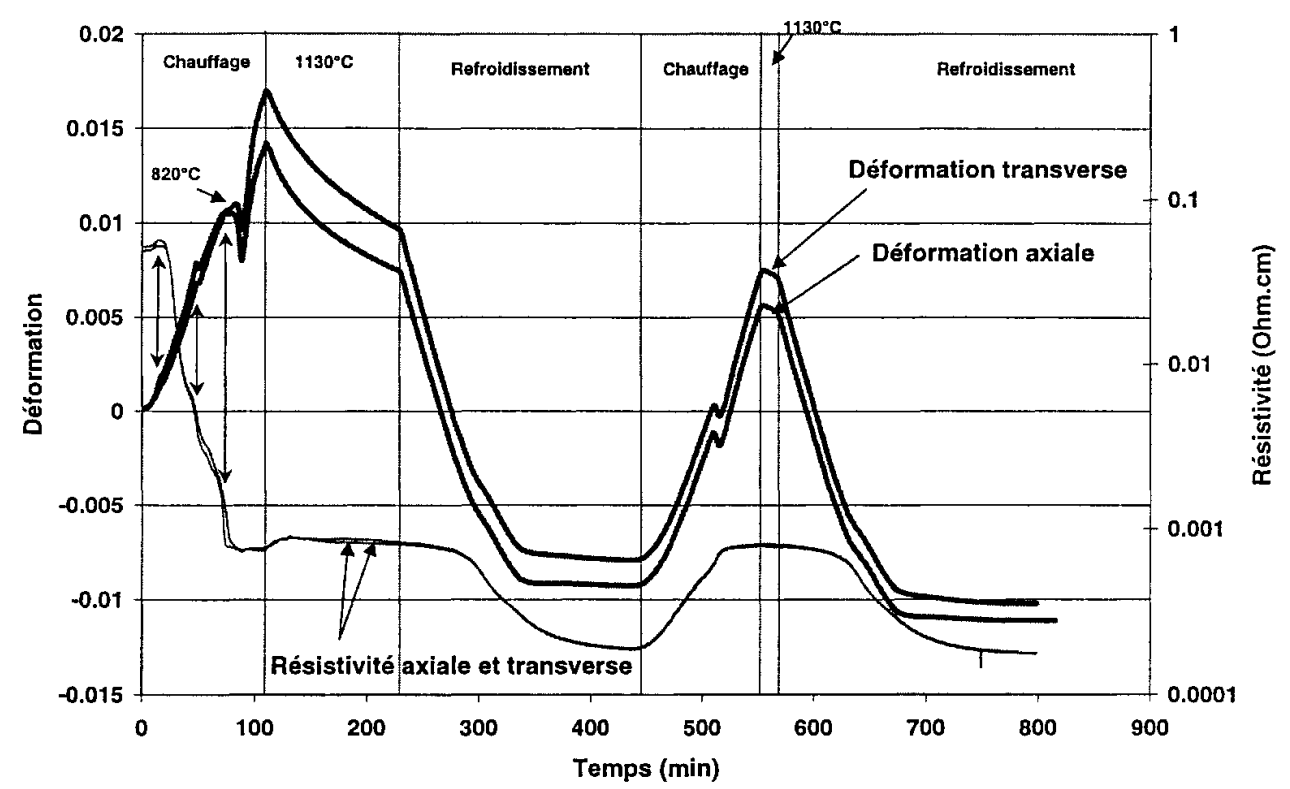

Figure 2 : Mesures de retrait et de résistivité pour des échantillons à $6.5 \mathrm{~g} / \mathrm{cm}^{3}$ pendant les deux cycles thermiques successifs.

Les mesures de résistivité sont reportées sur la même figure. Lors du premier cycle thermique, la résistivité décroît considérablement pendant la montée en température. Cette diminution est probablement liée au soudage des contacts interparticulaires formés pendant la compaction. Quelques minutes avant le palier isotherme, la résistivité est 100 fois plus faible qu'au début du cycle. Pendant le palier, alors qu'un retrait est observé, la résistivité ne décroît que faiblement. De plus, pour une telle pression de compaction, aucune anisotropie de résistivité n'est observée. Lors du second cycle, les évolutions de résistivité sont beaucoup plus faibles et quasiment réversibles. Ainsi, ces évolutions sont supposées intrinsèques au matériau. Nous supposons alors que les variations de résistivité qui apparaissent lors du second cycle sont négligeables devant celles du premier. Le matériau est supposé très proche de l'équilibre thermodynamique. Cet état sera appelé "état final" de frittage. Les résultats obtenus lors du second cycle seront utilisés comme références pour normaliser les données du premier cycle. Ainsi seuls les effets irréversibles dus au frittage seront mis en évidence.

La même expérience a été réalisée pour deux échantillons compactés à $750 \mathrm{MPa}$. Les mêmes tendances sont observées (Figure 3). Cependant, l'anisotropie en déformation est bien plus importante, à la fois pendant la montée et pendant le palier isotherme. Après refroidissement, le retrait est deux fois plus important dans la direction de compaction que dans la direction transverse. En ce qui concerne la résistivité électrique, il faut d'abord noter que la résistivité mesurée dans le sens axial au début du cycle est $20 \%$ plus faible que celle mesurée dans le sens transverse. Ce résultat peut se comprendre dans la mesure où la poudre a subi des contraintes plus fortes dans le sens axial que dans le sens transverse, ce qui se traduit probablement par une meilleure qualité de contact dans le sens axial. Cependant cet écart est rapidement résorbé lors de la montée en température. La tendance s'inverse même après le changement de phase. L'écart reste ensuite inchangé même après le second cycle. Jusqu'ici nous n'avons pas été en mesure d'expliquer ce phénomène qui devra être confirmé par de nouvelles expériences. 


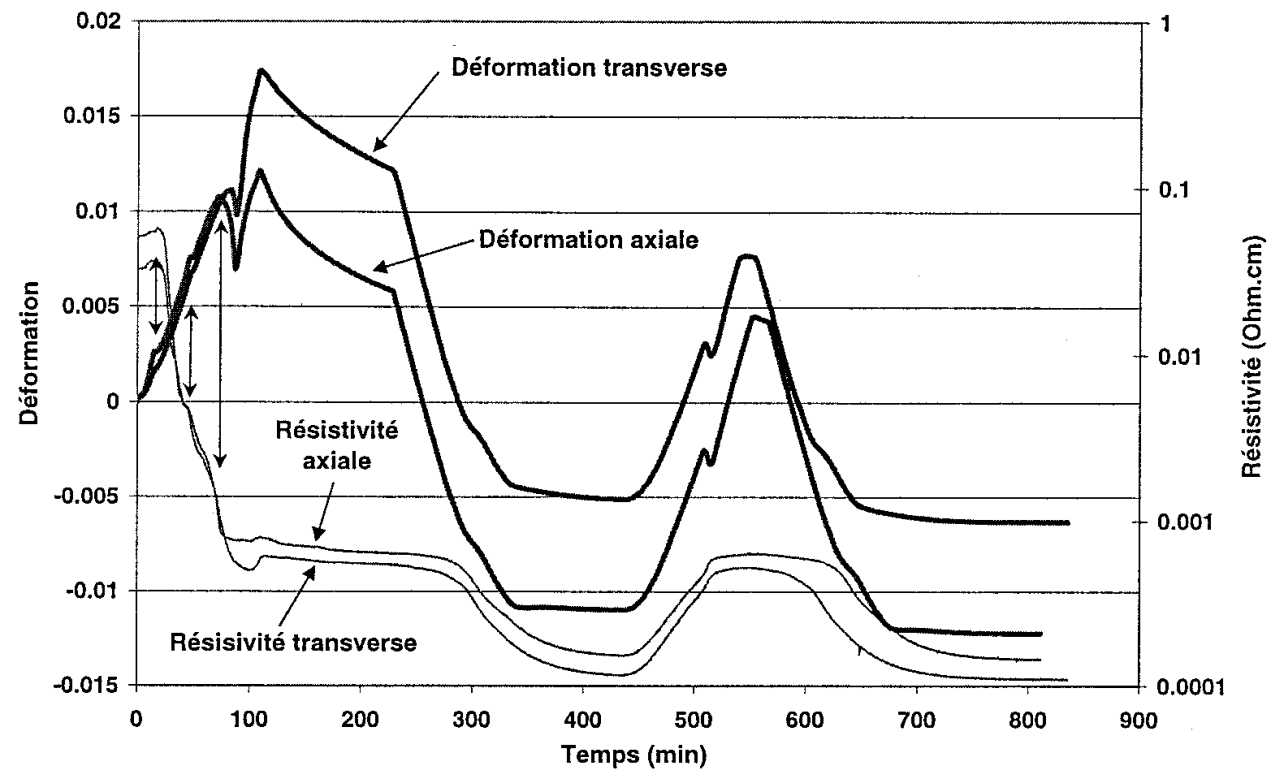

Figure 3 : Mesures de retrait et de résistivité pour des échantillons à $7.1 \mathrm{~g} / \mathrm{cm}^{3}$ pour les deux cycles thermiques successifs.

\section{MESURES DE VISCOSITE}

\subsection{Equipement}

Le principal cycle thermique utilisé pour ces mesures est dérivé d'un cycle industriel. Il comporte une rampe à $40^{\circ} \mathrm{C} / \mathrm{min}$, un palier isotherme d'une heure à $1130^{\circ} \mathrm{C}$ et un refroidissement rapide. Les échantillons utilisés sont des parallélépipèdes de $15 \times 3 \times 2 \mathrm{~mm}$. Pendant le cycle thermique, un essai de flexion est réalisé (figure 4). L'échantillon est placé sur deux appuis espacés de $12 \mathrm{~mm}$ et une tige d'alumine est positionnée en son milieu. Cette tige permet d'une part de mesurer la flèche de la poutre au cours du temps, d'autre part d'appliquer continûment une charge comprise entre 15 et $150 \mathrm{~g}$. Lors d'un tel essai la contrainte est maximale dans la direction de la longueur de la poutre. Ainsi, la viscosité déduite est principalement représentative de la viscosité du matériau dans la direction transverse. Par ailleurs, pour cet essai, le retrait dû au frittage naturel est négligeable devant la flèche.

Deux types d'échantillons ont été testés. D'abord des échantillons « à vert » donnant accès à des informations sur l'évolution de la viscosité induite par le frittage. D'autre part, des échantillons préalablement frittés pendant 5 heures à $1130^{\circ} \mathrm{C}$. Ces derniers sont supposés avoir atteint l'état final de frittage et seront utilisés pour normaliser les résultats du premier essai.

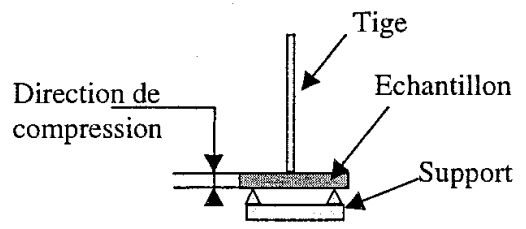

Figure 4 : Schéma du dispositif de flexion 


\subsection{Résultats des mesures de viscosité}

Classiquement, la mesure de viscosité en cours de frittage se fait par application de courtes périodes de chargement en compression $[5,11]$. La viscosité est alors déduite du saut de vitesse de déformation induit par la charge. Dans ce cas l'état de contrainte est homogène et l'interprétation de l'essai est donc simple. En flexion, l'état de contrainte est plus complexe mais on peut appliquer des contraintes beaucoup plus importantes avec la même charge. Par exemple, pour une charge de $150 \mathrm{~g}$, la contrainte maximale sera d'environ .6 MPa pour la flexion alors qu'il est difficile de dépasser $0,2 \mathrm{MPa}$ en compression en gardant une section d'éprouvette raisonnable. Pour les échantillons «à vert », les deux méthodes peuvent être utilisées. Pour les échantillons dans l'état final, la densité est très grande et par conséquent l'effet d'une faible contrainte est trop petit pour que l'on puisse mesurer la viscosité avec une précision suffisante. Dans ce cas la technique de mesurer en flexion est particulièrement bien adaptée. Les figures 5 et 6 montrent l'évolution de la flèche de deux échantillons, l'un «à vert » (a) et le deuxième dans l'état final (b).

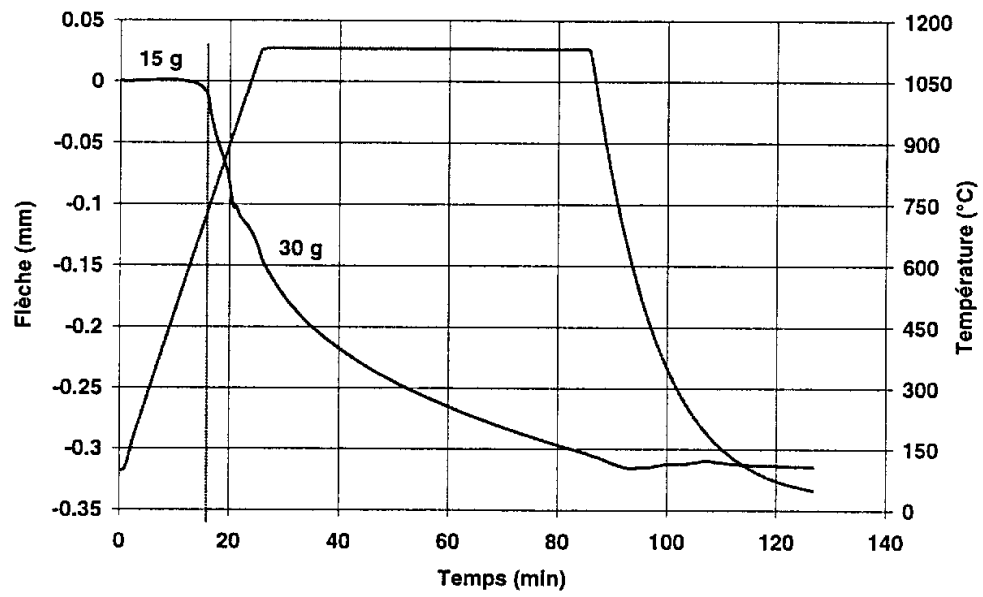

Figure 5 : Flèche mesurée en flexion pour un échantillon « à vert »

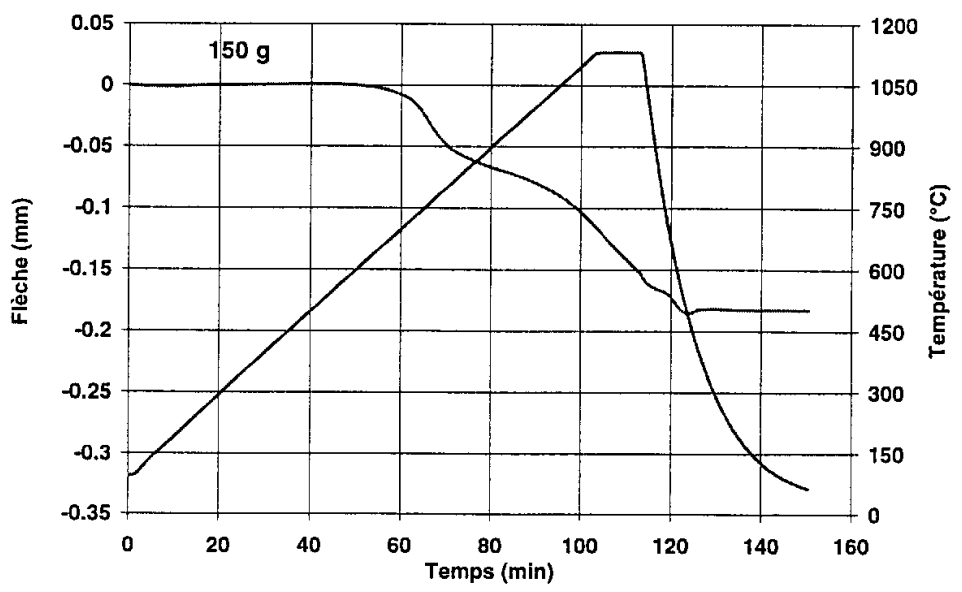

Figure 6 : Flèche mesurée en flexion pour un échantillon dans l'état final 
Au début du cycle, l'échantillon (a) a une très faible résistance mécanique. Pour minimiser le risque d'endommagement, une charge relativement faible de $15 \mathrm{~g}$ est appliquée jusqu'à $700^{\circ} \mathrm{C}$. Par la suite, pour améliorer la précision, une charge de $30 \mathrm{~g}$ est appliquée jusqu'à la fin du cycle. On observe que la flèche commence à évoluer vers $600^{\circ} \mathrm{C}$. Ensuite, la flèche augmente continûment, fortement pendant la rampe et plus lentement pendant le palier isotherme. La microstructure de l'échantillon (b) est supposée stable, par conséquent, son comportement ne doit dépendre que de la température. Pour augmenter la précision sur la température, l'échantillon (b) est soumis à un cycle thermique comportant une rampe à $10^{\circ} \mathrm{C} / \mathrm{min}$. La charge appliquée est de $150 \mathrm{~g}$. Bien que la charge soit 5 fois plus grande que celle appliquée sur l'échantillon (a), la flèche reste plus faible. Il est donc clair que la viscosité est très supérieure.

A partir de ces résultats, on peut calculer la viscosité à tout moment du cycle thermique à partir d'une analogie élastique/visqueuse. En effet, selon la théorie des poutres élastiques, la flèche maximale d'une poutre reposant sur 2 appuis et soumise à une force ponctuelle $F$ en son centre (flexion 3 points) est :

$$
f=\left[\frac{1}{4} \frac{\mathrm{Fl}^{3}}{\mathrm{bh}^{3}}\right] \frac{1}{\mathrm{E}}
$$

Où $b, h, 1$ sont respectivement la largeur, la hauteur, la longueur de la poutre et $E$ le module d'Young. Dans le cas d'un comportement visqueux linéaire de viscosité $\mathrm{v}$, on suppose qu'un incrément de flèche df pendant un incrément de temps dt peut être estimé en remplaçant dans l'équation (2) E par v/dt. Ainsi, la viscosité est donnée tout au long du cycle thermique par :

$$
v(t)=\frac{F 1^{3}}{4 b h^{3}(d f / d t)}
$$

Cette méthode a été validée précédemment [12].

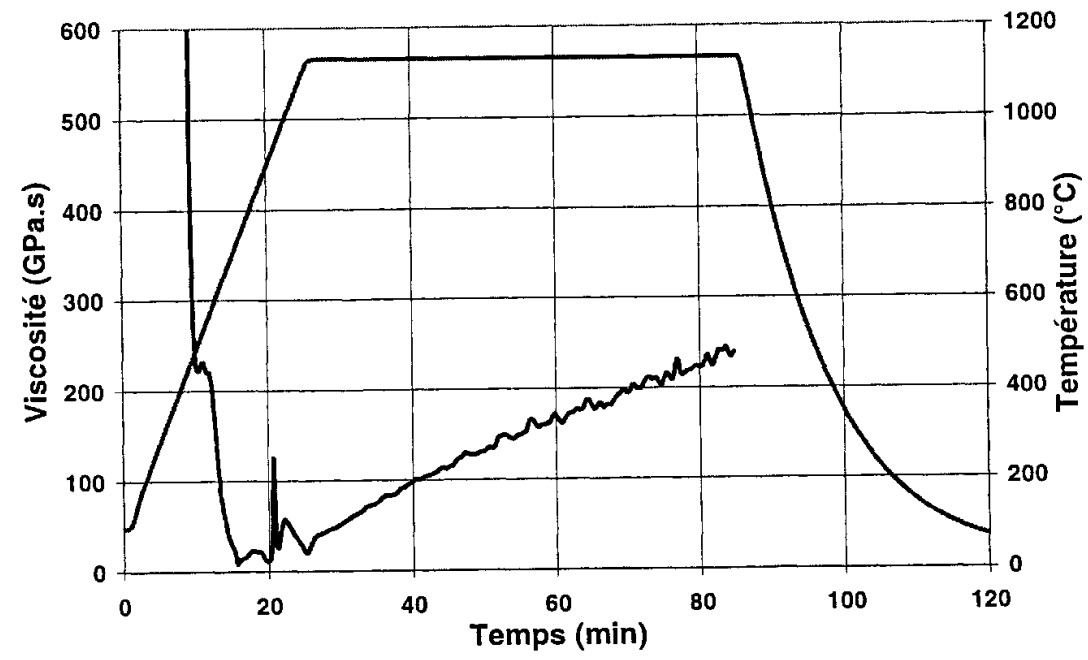

Figure 7. Viscosité de l'échantillon (a) mesurée en flexion au cours d'un cycle thermique classique 
Les résultats obtenus pour l'échantillon (a) sont présentés sur la figure 7. Lors de la montée en température, la valeur de la viscosité est le produit de la compétition de deux effets opposés. D'une part l'augmentation de la température qui tend à diminuer la viscosité du matériau, d'autre part, le frittage qui tend à souder les particules entre elles et donc à augmenter la viscosité. On observe globalement une diminution de la viscosité jusqu'à une valeur minimale de $10 \mathrm{GPa} . \mathrm{s}$. Vers $900^{\circ} \mathrm{C}$, la transformation de phase $\alpha \rightarrow \gamma$ du fer produit apparemment une stabilisation de la viscosité alors que la température augmente. Pendant le palier à $1130^{\circ} \mathrm{C}$, la viscosité augmente régulièrement ce qui est vraisemblablement la conséquence des diverses évolutions microstructurales du matériau (diffusion des éléments d'additions, éventuel grossissement des grains). Après une heure la viscosité atteint $240 \mathrm{GPa}$.s. La viscosité de l'échantillon (b) a été obtenue par la même méthode. Les résultats seront utilisés pour normaliser les données obtenues lors du premier essai.

\section{RESULTATS NORMALISES}

Sur la figure 8 sont présentés les résultats de viscosité et de résistivité normalisés par les valeurs obtenues sur des échantillons dans l'état final. Pour chaque propriété physique, on définit ainsi un paramètre adimensionnel qui ne représente que les évolutions irréversibles survenant en cours de frittage. Ces paramètres d'avancement du frittage ont une valeur nulle avant frittage et une valeur de 1 dans l'état final On observe clairement que les cinétiques d'évolution des deux paramètres sont très différentes. En effet, concernant le paramètre lié à la résistivité, $98 \%$ de l'évolution est effectuée avant même l'arrivée au palier isotherme. Le long du palier isotherme l'augmentation est très lente. Concernant le paramètre lié à la viscosité, après une augmentation rapide au tout début du cycle, l'évolution devient relativement lente pour le reste du cycle. Pendant le changement de phase la courbe est très perturbée mais évolue globalement assez peu. Ensuite le long du palier isotherme une lente augmentation peut être observée, le paramètre atteignant après 1 heure la valeur de 0.13 , soit $13 \%$ de sa valeur finale.

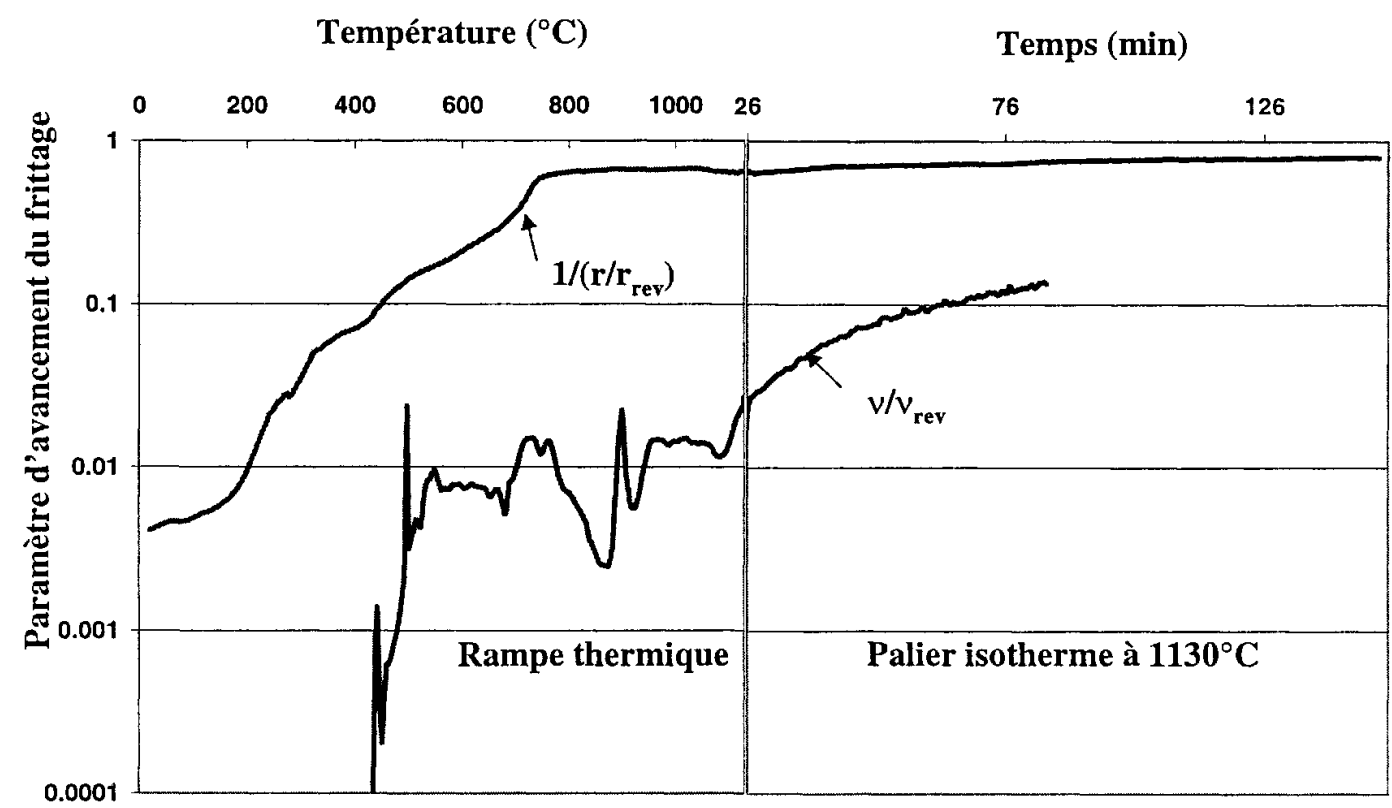

Figure 8 : Paramètre d'avancement du frittage au cours du cycle thermique. 


\section{CONCLUSION ET DISCUSSION}

Un dispositif expérimental innovant été développé pour mesurer in situ simultanément le retrait et la résistivité électrique d'un matériau au cours d'un cycle thermique. Par ailleurs, une méthodologie expérimentale a été définie pour mesurer la viscosité en flexion d'un matériau en cours de frittage moyennant une analogie élastique/visqueuse. Ces deux expériences ont été réalisées sur des comprimés d'acier Distaloy AE.

Un paramètre adimensionnel a été déduit de ces deux essais. Malgré les incertitudes de mesures les deux paramètres semblent pertinents pour décrire les évolutions irréversibles du matériau et par conséquent l'avancement du frittage. Le paramètre déduit de la résistivité est probablement corrélé au soudage interparticulaire. En effet, la formation de pont interparticulaire est un phénomène souvent considéré comme instantané en début de frittage, ce qui peut expliquer la très forte augmentation qui apparaît très tôt dans le cycle thermique. Ensuite l'augmentation est très lente sur le palier, ce qui est logique compte tenu du fait que le retrait est très faible pour ce type de matériau. Le paramètre déduit de la viscosité augmente beaucoup plus lentement en cours de frittage. L'augmentation est observable à la fois pendant le chauffage et le palier isotherme. Sa valeur est multipliée par 4 après la première heure de frittage mais reste faible. Les variations de ce paramètre sont vraisemblablement représentatives de l'évolution microstructurale du matériau liée notamment à la diffusion des éléments d'additions.

Les deux paramètres d'avancement du frittage pourraient être utilisés pour une description macroscopique du frittage. L'interprétation physique de l'évolution de chaque paramètre fait actuellement l'objet d'études microstructurales, soit par microscope électronique à balayage, soit grâce à une technique d'imagerie 3D plus récente et très prometteuse, la microtomographie aux rayons X [13].

\section{REFERENCES}

1 V.V. Skorokhod, Poroskova Metall., 2, 1961, 14-20.

2 C.R. Reid, Mechanics of Gramular Materials and Powder Systems, Proceeding of ASME., 37, 1992, 19-27.

3 C.R. Reid, Powder Tech., 81, 287, 1994.

4 E.A. Olevsky, Mat. Sci. Eng. Report, R23(2), 1998.

5 P. Z. Cai, G. L., Messing, D. J. Green, J. Am. Ceram. Soc., 80, 1997, 445-452.

6 O. Lame, D. Bouvard and H. Wiedemann, Powder Met., 45, No 1, 2002.

7 A. Simchi, H. Danninger, Powder Met., 43, No3, 2000, 209.

8 A. Simchi, et al., Powder Met., 43, No 3, 2000, 219.

9 A. Simchi, et al., Powder Met., 44, No 2, 2001, 148-156.

10 L.P. Lefebre, G Pleizier, Y. Deslandes, Powder Met., 44, No 3, 2001, 259-266.

11 O.Gillia, C.Josserond, D. Bouvard, Acta Mater., 49, No 8, 2001, 1413-1420.

12 O. Lame and D Bouvard, Proceeding of the European Congress on Powder Metallurgy (Euro PM2001), EPMA Shrewsbury UK, Nice, 2001.

13 O. Lame, D. Bellet, M. Di Michiel et D. Bouvard, Proceeding of the European Congress on Powder Metallurgy (Euro PM2001), EPMA Shrewsbury UK, Nice, 2001. 